\title{
An inverse model for locating skin tumours in 3D using the genetic algorithm with the Dual Reciprocity Boundary Element Method
}

\section{Abstract}

Here, the Dual Reciprocity Boundary Element Method is used to solve the 3D Pennes Bioheat Equation, which together with a Genetic Algorithm, produces an inverse model capable of obtaining the location and the size of a tumour, having as data input the temperature distribution measured on the skin surface.

Given that the objective function, which is solved inversely, involves the DRBEM (Dual Reciprocity Boundary Element Method) the Genetic Algorithm in its usual form becomes slower, in such a way that it was necessary to develop functions based the solution history in order that the process becomes quicker and more accurate.

Results for 8 examples are presented including cases with convection and radiation boundary conditions. Cases involving noise in the readings of the equipment are also considered.

This technique is intended to assist health workers in the diagnosis of tumours.

\section{Keywords}

Inverse method, Genetic Algorithm, Dual Reciprocity Boundary Element Method, Tumour, Pennes Bioheat Equation.

\section{Fabrício Ribeiro Bueno*, a and Paul William Partridge ${ }^{b}$ \\ ${ }^{a}$ Programa de pos-graduação em Estru- turas e Construção Civil, Universidade de Brasília, 70910-900 Brasília-DF, Brazil. \\ ${ }^{b}$ Professor, Departamento de Eng. Civil e Ambiental, Universidade de Brasília, 70910-900 Brasília-DF, Brazil.}

Received 05 Feb 2012

In revised form 15 Mar 2013

*Author email: eng.frb@gmail.com

\section{INTRODUCTION}

There exist various techniques for the localization of tumours: Invasive techniques, which need surgery and non-invasive techniques, such as ultra sound, MRI and thermal methods. Amongst the non-invasive techniques, thermal methods stand out as economic and safe (Deng and Liu, 2004).

A steady-state mathematical model based on the Bioheat Equation developed by Pennes (1948), simulating heat transfer throughout the human body, was developed by Wissler (1961). These results were compared with experimental data and were considered satisfactory.

Liu and $\mathrm{Xu}$ (2000) investigated thermal behaviour in live tissue subject to heating in different ways. One of the means of heating studied is the local heating of the skin due to the presence of skin tumours. For this analysis Liu and Xu used a model based on the Dual Reciprocity Boundary 
Element Method, to obtain the temperature distribution at the skin surface. The Boundary Element Method (BEM) is a numerical technique that requires only discretization of the boundary thus reducing the quantity of data necessary to run a problem when comparing to another methods. DRBEM is essentially a generalized way of constructing particular solutions that can be used to solve non-linear and time-dependent problems as well as to represent any integral source distribution (Partridge et al, 1992).

Considering these observations Partridge and Wrobel (2007) developed an inverse procedure using the Genetic Algorithm, through which the size and location of a skin tumour could be obtained from temperatures measured at the skin surface in a two dimensional model. These results were later improved by Wrobel et al (2009) who implemented in the algorithm the capability of locating tumours with a circular geometry.

The Genetic Algorithm is an optimization method which in the references cited has shown itself to be viable for diagnoses based on thermal information. One of its advantages is that it is unnecessary to supply data on an initial position of the tumour. In addition it is not necessary to calculate sensitivities or search directions.

Here an inverse technique is used, which instead of starting from a tumour for which the size and position are known and the temperature distribution which this provokes on the skin surface calculated, starts from a distribution of temperatures measured in practice by equipment having thermometers created for this purpose, and from this the size and position of the tumour is obtained. The tumour models considered here are in $3 \mathrm{D}$, and not in plane form as in the previous studies, Chan (1992), Deng and Liu (2000), Liu and Xu (2000) and Deng and Liu (2004), Partridge and Wrobel, (2007) and Wrobel et al (2009). In this way the results obtained are more complete and satisfactory.

\section{PENNES BIOHEAT EQUATION}

According to Pennes (1948) the rate of transfer of heat between blood and tissue is proportional to the product of the rate flow of blood and the difference between the temperature of arterial blood and the temperature of local tissue. Thus the bioheat equation was written as follows:

$$
h_{b}=\omega_{b} \rho_{b} c_{b}(1-\kappa)\left(T_{a}-T\right)
$$

Where $h_{b}$ is the rate of transfer of heat per unit volume of tissue, $\omega_{b}$ is the rate of flow of blood per unit volume of tissue, $\rho_{b}$ is blood density, $c_{b}$ is the specific heat of blood, $\kappa$ is a coefficient which takes into account the incomplete thermal equilibrium between tissue and blood, $T_{a}$ is the arterial blood temperature and $T$ is the local tissue temperature. Pennes considered that $\kappa$ varies between 0 and 1 , however took $\kappa=0$ for his theoretical curves as has been done by researchers since (Wissler, 1998).

The thermal energy balance for perfusion in the tissue is expressed in the following form:

$$
\rho c \frac{\partial T}{\partial t}=k \nabla^{2} T+\omega_{b} \rho_{b} c_{b}\left(T_{a}-T\right)+Q
$$

Latin American Journal of Solids and Structures 10(2013) $1061-1079$ 
Which can be rewritten as:

$$
\frac{\rho c}{k} \frac{\partial T}{\partial t}=\nabla^{2} T+\frac{\omega_{b} \rho_{b} c_{b}}{k}\left(T_{a}-T\right)+\frac{Q}{k}
$$

Where $\rho, c$ and $k$ represent respectively density, specific heat and thermal conductivity of the tissue and $Q$ is the rate of production of metabolic heat per unit volume of tissue.

Eqn. (3) is subject to the usual boundary conditions for thermal problems:

$1^{\mathrm{o}}$ ) temperature prescribed $T=\bar{T}$;

$2^{\mathrm{O}}$ ) heat flux prescribed $q=\bar{q}$;

$\left.3^{\mathrm{o}}\right)$ convection $q=-h_{0}\left(T-T_{0}\right)$, where $h_{0}$ is the coefficient of heat transfer and $T_{0}$ is the temperature of the surrounding fluid;

$4^{\mathrm{o}}$ ) radiation $q=-\sigma \varepsilon\left(T_{r}^{4}-T^{4}\right)$, where $\sigma$ is the Stefan-Boltzman constant and $\varepsilon$ is the radiative interchange factor between the surface and the exterior ambient temperature $T_{r}$.

For an analysis at a given instant, eqn. (3) should be written in its steady state form, thus becoming:

$$
\nabla^{2} T=-\frac{\omega_{b} \rho_{b} c_{b}}{k}\left(T_{a}-T\right)-\frac{Q}{k}
$$

Including the constants in (4) one has:

$$
\nabla^{2} T=-c_{1}\left(T_{a}-T\right)-c_{2}
$$

Where $c_{1}$ and $c_{2}$ are written as:

$$
\begin{gathered}
c_{1}=\frac{\omega_{b} \rho_{b} c_{b}}{k} \\
c_{2}=\frac{Q}{k}
\end{gathered}
$$

Carrying out the multiplication eqn.(5) can also be written as:

$$
\nabla^{2} T=-c_{1} T_{a}+c_{1} T-c_{2}
$$

Given that $T_{a}$ is constant, eqn. (6) reduces to:

$$
\nabla^{2} T=c_{3}+c_{1} T
$$


Where:

$$
c_{3}=-c_{1} T_{a}-c_{2}
$$

Eqn.(7), considering only constant $c_{3}$, is a Poisson equation, and when $c_{1} T$ is considered (7) is a Helmholtz equation. The first term is a function of space, and does not include the problem variable, and the second is dependent on the variable $T$. Here these equations are solved using DRBEM

The values of the parameters necessary to calculate $c_{1}$ and $c_{3}$ in eqn. (7) were taken from Liu and $\mathrm{Xu}(2000)$ as being: $\rho_{b}=1000 \mathrm{~kg} / \mathrm{m}^{3}, c_{b}=4000 \mathrm{~J} /\left(\mathrm{kg}^{\circ} \mathrm{C}\right)$ and $k=0.5 \mathrm{~W} /\left(\mathrm{m}^{\circ} \mathrm{C}\right)$, in addition, $\omega_{b}=0.0005 \mathrm{ml}_{b} /\left(\mathrm{ml}_{t} s\right)$ and $Q=420 \mathrm{~J} /\left(\mathrm{m}^{3} s\right)$ for healthy tissue, whilst for tissue with a tumour $\omega_{b}=0.002 m l_{b} /\left(m l_{t} s\right)$ and $Q=4200 \mathrm{~J} /\left(\mathrm{m}^{3} s\right)$, in which the subscripts $b$ and $t$ represent blood and tissue, respectively. The temperature of arterial blood is $T_{a}=37^{\circ} \mathrm{C}$.

\subsection{Application of DRM to the Bioheat Equation}

These equations, Poisson and Helmholtz, are solved separately in Brebbia and Dominguez (1989), where the fundamental solution for isotropic media in $3 \mathrm{D} u^{*}=1 /(4 \pi r)$, is employed to deal with the left hand side of eqn. (7) and the right hand side is taken to the boundary using the Dual Reciprocity Method as in Partridge, et al (1992), leading to the following system of equations

$$
H u-G q=(H \hat{u}-G \hat{q}) F^{-1} c_{3}
$$

For the Poisson equation, and

$$
H u-G q=(H \hat{u}-G \hat{q}) F^{-1} u c_{1}
$$

For the Helmholtz equation.

In this way the matrix equation for the solution of the bioheat equation is

$$
H u-G q=(H \hat{u}-G \hat{q}) F^{-1} c_{3}-(H \hat{u}-G \hat{q}) F^{-1} u c_{1}
$$

Where the matrix $F$ is calculated from the approximation function used in the method. Putting $S=(H \hat{u}-G \hat{q}) F^{-1}$ one obtains

$$
H u-G q=S c_{3}-S c_{1} u
$$

Reorganizing the matrices

Latin American Journal of Solids and Structures 10(2013) 1061 - 1079 


$$
\left(H+S c_{1}\right) u=G q+S c_{3}
$$

Applying the boundary conditions produces the usual system of equations:

$$
A x=y
$$

\subsection{Application of the convection and radiation boundary conditions}

One can consider the boundary condition $q=\bar{q}$ when the skin surface is bandaged. Otherwise it is more realistic to consider convection and radiation boundary conditions, Liu e Xu (2000). The convection and radiation boundary conditions are given by, respectively:

$$
q=-h\left(T-T_{0}\right)
$$

and

$$
q=-\sigma \varepsilon\left(T^{4}-T_{r}^{4}\right)
$$

Where $q=K \partial T / \partial n, \bar{T}$ and $\bar{q}$ are known values, $h$ is the coefficient of heat transfer, $T_{0}$ is the average temperature of the surrounding fluid, $\sigma$ is the Stefan-Boltzmann constant, $\mathcal{E}$ is the radiative interchange factor between the surface and the exterior environment at temperature $T_{r}$

A case considering convection and radiation together can be written:

$$
-q=\sigma \varepsilon\left(T^{4}-T_{r}^{4}\right)+h\left(T-T_{0}\right)
$$

One can expand the previous equation as follows:

$$
-q=\sigma \varepsilon\left(T^{2}+T_{r}^{2}\right)\left(T+T_{r}\right)\left(T-T_{r}\right)+h\left(T-T_{0}\right)
$$

The coefficient of apparent heat transfer by radiation for small variations in ambient temperature is $h_{r}=\sigma \varepsilon\left(T^{2}+T_{r}^{2}\right)\left(T+T_{r}\right)$. Under normal conditions $h_{r}=6,19 \mathrm{~W} / \mathrm{m}^{2}$ (Liu and $\mathrm{Xu}$, 2000). In this way:

$$
-q=h_{r}\left(T-T_{r}\right)+h\left(T-T_{0}\right)
$$

Under the same conditions given above of small variations of ambient temperature for radiation and convection it is possible to consider $T_{r}=T_{0}$ which is equivalent to the ambient temperature in question, here taken as $T_{a}=25^{\circ} \mathrm{C}$. In this way eqn. (18) can be written as:

$$
-q=\left(h_{r}+h\right)\left(T-T_{a}\right)
$$


According to Liu and $\mathrm{Xu}$ (2000), for a preliminary analysis, $h_{e f}=h_{r}+h=10 \mathrm{~W} / \mathrm{m}^{2}$. In this way the boundary condition for the elements which are in contact with the external environment is simplified to:

$$
q=-h_{e f}\left(T-T_{a}\right)
$$

This value of external temperature is lower than the temperature on the skin surface, which indicates that there will be a reduction in the values of observed temperature.

\subsection{Approximation functions and augmentation functions}

Bueno and Partridge (2011) considered 3D problems which simulate skin surface temperature using DRBEM with constant triangular boundary elements and tested the use of the following approximation functions $f=r, f=r^{3}, f=1+r, f=1+r^{3}, f=r^{2} \log r$, and $f=r^{4} \log r$, for various cases together with the linear augmentation functions $1, x, y$ and $z$, and concluded that the Dual Reciprocity Method produces the best results if the approximation function $f=1+r$ is used together with the linear augmentation functions $1, x, y$ and $\mathrm{z}$.

\section{MODELLING THE DIRECT PROBLEM}

\subsection{Rectangular Model in 3D}

The 3D model was constructed in such a way as to permit comparison with the $2 \mathrm{D}$ results obtained in previous work, the model has thus the parallelepiped form ABCDEFGH (Fig. 1) representing a sample of skin with a rectangular surface of $0.08 \times 0.08 \mathrm{~m}^{2}$ and a depth of $0.03 \mathrm{~m}$.

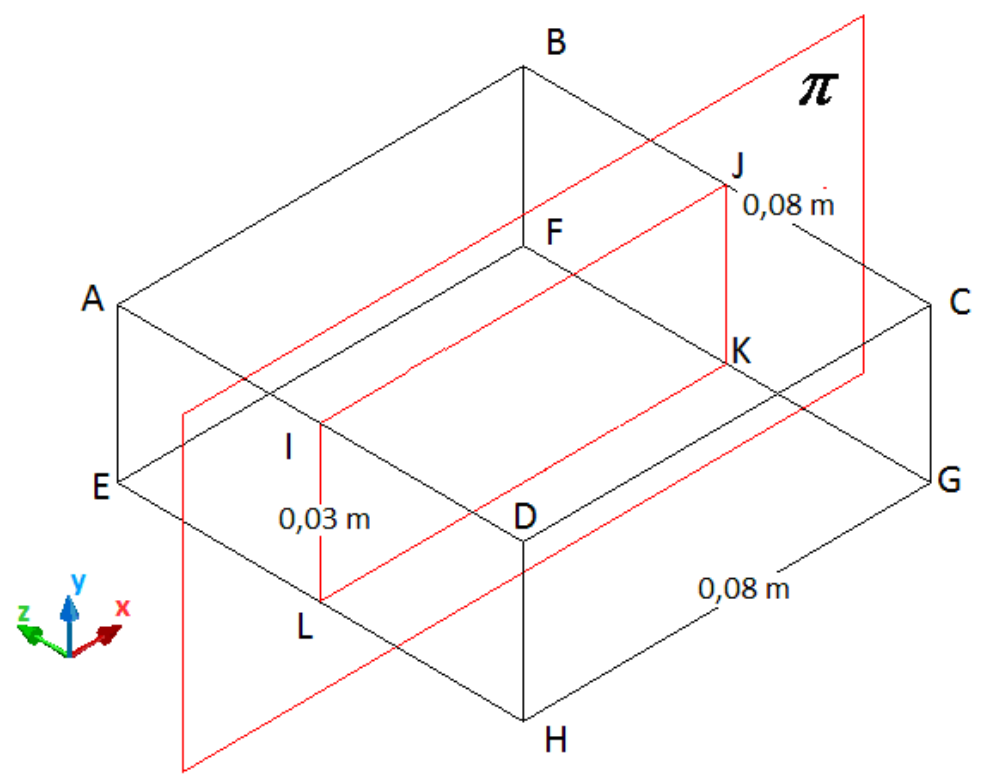

Figure 1 Obtaining the simplified 2D model from the 3D model considered here. 
Where the $\pi$ plane cuts the 3D model one obtains the 2D model studied in previous work

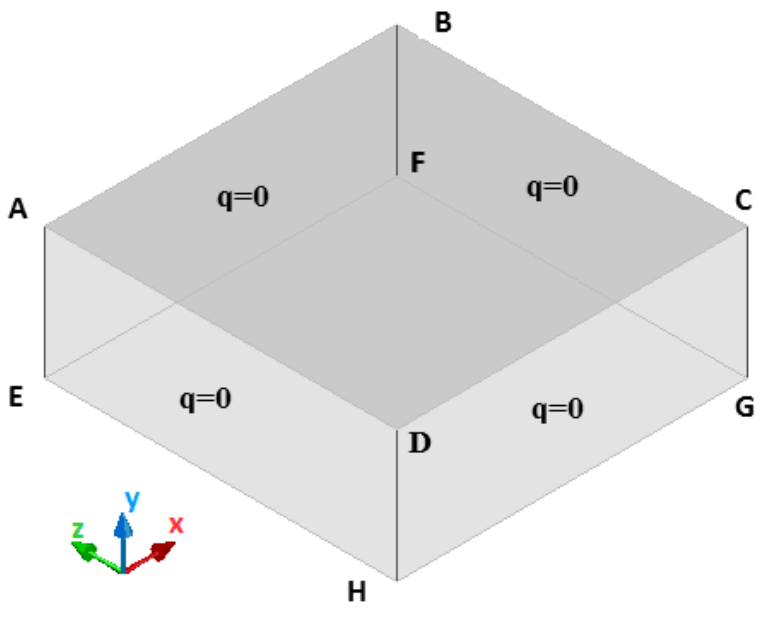

a)

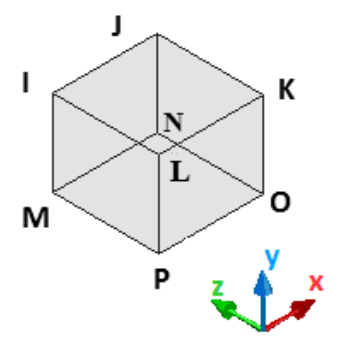

b)

Figure 2 a) Model of healthy tissue in 3D b) 3D rectangular model of tumour.

Fig. 2 (a) shows the 3D representation of the skin tissue delimited by the boundary of the parallelepiped formed by the points $\mathrm{A}, \mathrm{B}, \mathrm{C}, \mathrm{D}, \mathrm{E}, \mathrm{F}, \mathrm{G}$ and $\mathrm{H}$ defined now as boundary $\Gamma_{2}$. In fig. 2 (b), delimited by the points I, J, K, L, M, N, O and P, one has the 3D model of a tumour of rectangular geometry now called boundary $\Gamma_{1}$. This latter will be in some position inside $\Gamma_{2}$.

Considering boundary $\Gamma_{2}$, the face EFGH is an internal surface and is considered to be at a constant temperature $T=37^{\circ} \mathrm{C}$ (internal body temperature). The limit ABCD is an external skin surface and can have a boundary condition $q=0$ if it is considered that the skin is bandaged. Another more realistic boundary condition for ABCD is to consider convection with the external environment. All the other limits of $\Gamma_{2}$ are artificial internal boundaries where the skin sample in question is in contact with other areas of skin and for these cases the boundary condition $q=0$ is considered. The boundary $\Gamma_{1}$ is considered to be a tumour internal to the boundary $\Gamma_{2}$ where the boundary conditions are that the temperature at a node on $\Gamma_{1}$ is equal to the temperature at the corresponding node on $\Gamma_{2}\left(T_{1}=T_{2}\right)$ and the exchange of heat done between the two regions is $q_{1}=-q_{2}$.

The discretization into boundary elements was done using 288 constant triangular elements on the $\Gamma_{2}$ boundary and 48 constant triangular elements on the $\Gamma_{1}$ boundary. This kind of elements used was chosen for two basic reasons: 1) It was desired to compare results with the $2 \mathrm{D}$ case from Partridge and Wrobel (2007); 2) It was desired to reduce processing time, which due to the combination of the Genetic Algorithm and Dual Reciprocity starred quite high and was reduced to about 20 minutes for one program run. 
Initially a rectangular tumour with dimensions $0.02 \times 0.02 \times 0.01 \mathrm{~m}^{3}$ and with its centre located at coordinates $(0.00 ; 0.00 ; 0.01)$ corresponding to the coordinates $\mathrm{x}, \mathrm{z}$ and $\mathrm{y}$ respectively, as shown in fig. 3 .

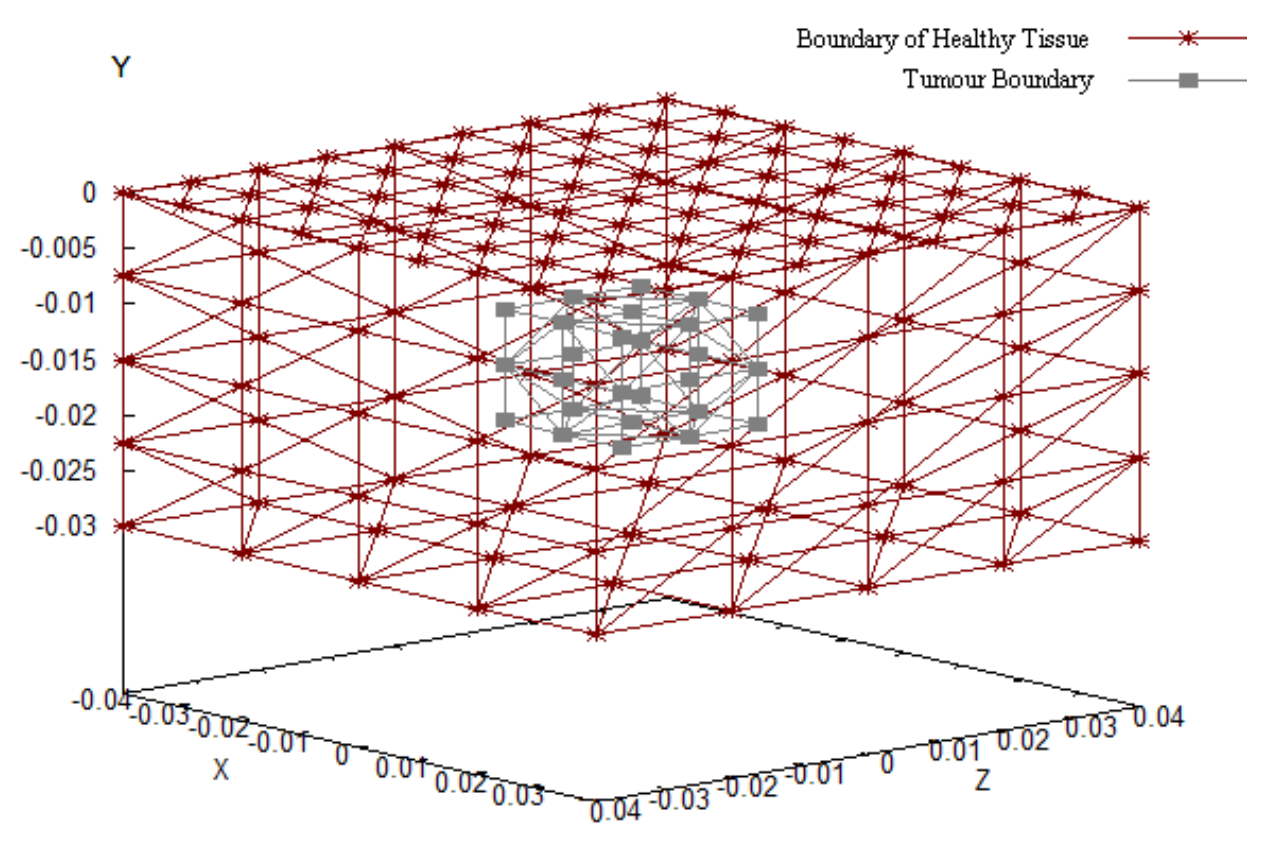

Figure 3 Geometry discretized using constant triangular boundary elements.

In order to find the temperature distribution on the surface ABCD of the tissue (Figs. 1 and 2 ) the Pennes Bioheat equation is solved using the Dual Reciprocity Boundary Element Method as described above.

\subsection{Spherical Tumour Models}

Using a similar reasoning, the spherical tumour model can use the same discretization of the healthy tissue (288 constant triangular elements), and the tumour is discretized using 48 constant triangular elements, (Fig. 5). 


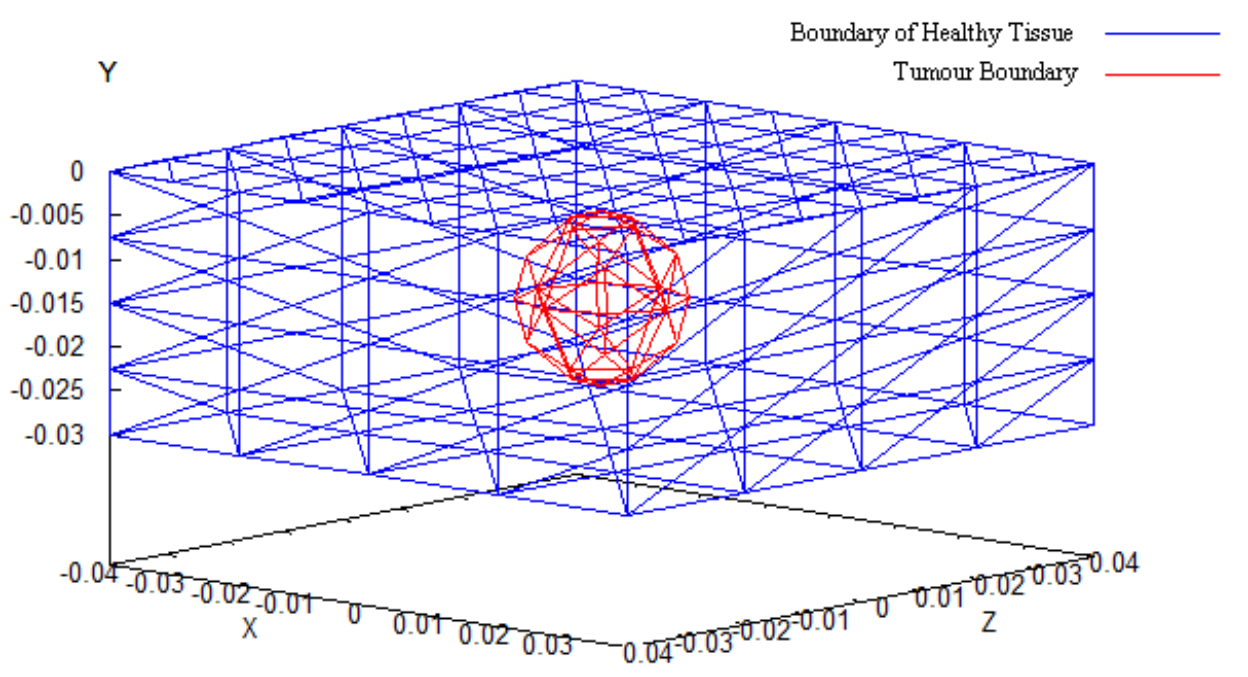

Figure 4 Spherical model of tumour discretized using 48 triangular elements

The values found for the spherical model and those found for the circular model studied by Wrobel et al (2009) are compared in table 1.

Table 1 Comparison of the results of the 3D model with those of the 2D model.

\begin{tabular}{|c|c|c|c|}
\hline \multirow{2}{*}{$\begin{array}{c}\text { Position } \\
X\end{array}$} & Reference & \multicolumn{2}{|c|}{ Sphere } \\
\cline { 3 - 4 } & $2 \mathrm{D}$ & Value & Difference \\
\hline-0.035 & 37.17256 & 37.02215 & $0.4 \%$ \\
\hline-0.030 & 37.17874 & 37.03496 & $0.4 \%$ \\
\hline-0.025 & 37.19029 & 37.04777 & $0.4 \%$ \\
\hline-0.020 & 37.20861 & 37.07328 & $0.4 \%$ \\
\hline-0.015 & 37.23475 & 37.09880 & $0.4 \%$ \\
\hline-0.010 & 37.26730 & 37.14506 & $0.3 \%$ \\
\hline-0.005 & 37.29831 & 37.19132 & $0.3 \%$ \\
\hline 0.000 & 37.31196 & 37.19152 & $0.3 \%$ \\
\hline 0.005 & 37.29831 & 37.19171 & $0.3 \%$ \\
\hline 0.010 & 37.26730 & 37.14593 & $0.3 \%$ \\
\hline 0.015 & 37.23475 & 37.10014 & $0.4 \%$ \\
\hline 0.020 & 37.20861 & 37.07535 & $0.4 \%$ \\
\hline 0.025 & 37.19029 & 37.05056 & $0.4 \%$ \\
\hline 0.030 & 37.17874 & 37.03900 & $0.4 \%$ \\
\hline 0.035 & 37.17256 & 37.02743 & $0.4 \%$ \\
\hline
\end{tabular}

The distribution of temperature on the surface for the spherical model with its centre at the coordinates $(0.00 ; 0.015 ; 0.00)$ and diameter $0.02 \mathrm{~m}$ is given in fig. 5. : 


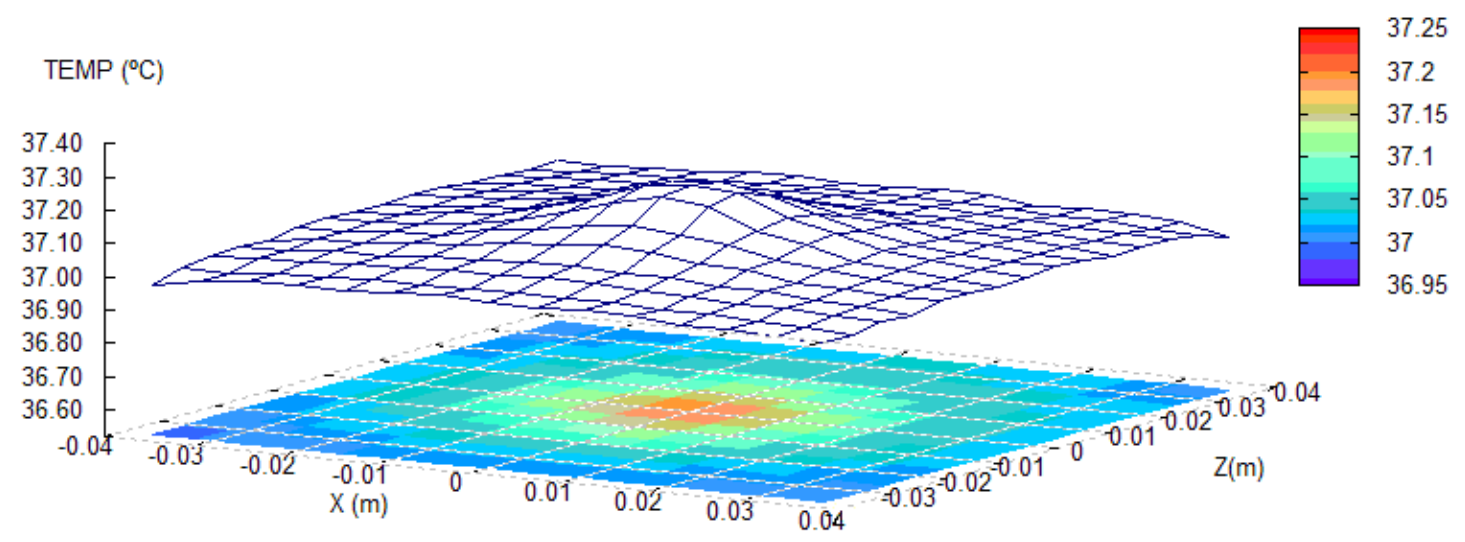

Figure 5 Temperature distribution for the spherical model .

\subsection{Convection and radiation boundary conditions}

In the following example a 3D model with a rectangular tumour of size $\left(0.02 \times 0.01 \times 0.02 \mathrm{~m}^{3}\right)$ centred on $(0.00 ; 0.01 ; 0.00)$, is considered, including convective boundary conditions as described above. The results are compared in Table 2 with those of a $2 \mathrm{D}$ convective model taken from Partridge and Wrobel (2007).

\section{TEMPERATURE DISTRIBUTION}

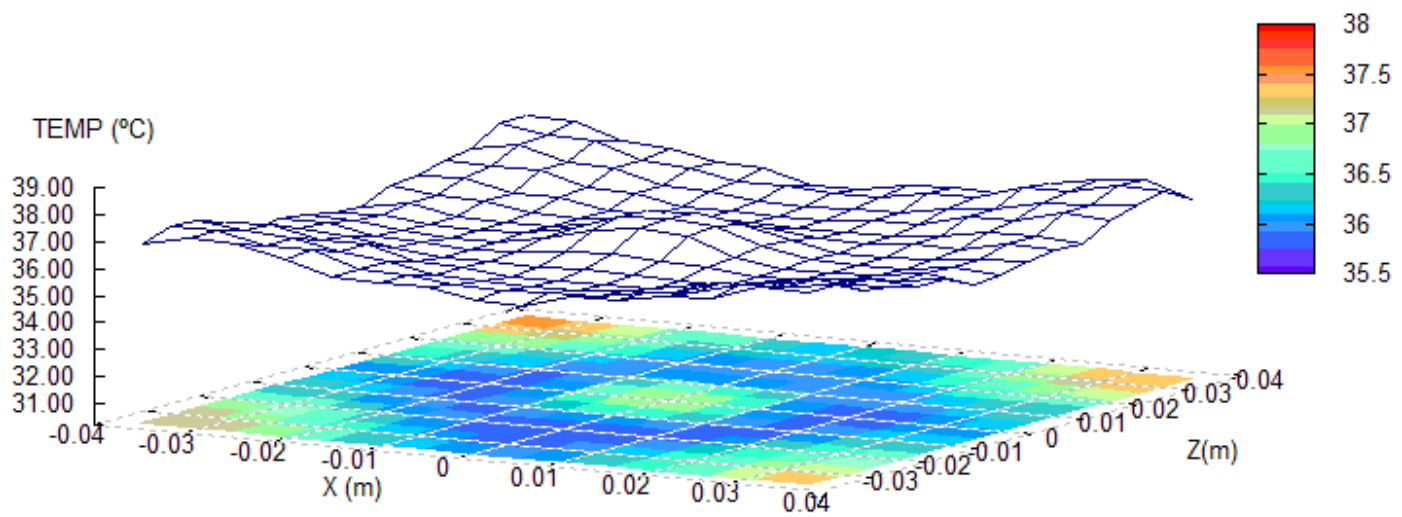

Figure 6 Distribution of temperature for a rectangular convective model. 
Table 2 Comparison of the results given by a 3D rectangular convective model with those of a $2 \mathrm{D}$ rectangular convective model.

\begin{tabular}{|c|c|c|c|}
\hline \multirow{2}{*}{$\begin{array}{c}\text { Position } \\
X\end{array}$} & Reference & \multicolumn{2}{|c|}{ 3D Model } \\
\cline { 3 - 4 } & 2D & Value & Difference \\
\hline-0.035 & 36.4937 & 36.0609 & $1.2 \%$ \\
\hline-0.030 & 36.4108 & 35.9834 & $1.2 \%$ \\
\hline-0.025 & 36.4216 & 35.9059 & $1.4 \%$ \\
\hline-0.020 & 36.4870 & 35.9406 & $1.5 \%$ \\
\hline-0.015 & 36.5951 & 35.9753 & $1.7 \%$ \\
\hline-0.010 & 36.7295 & 36.4219 & $0.8 \%$ \\
\hline-0.005 & 36.8390 & 36.8685 & $-0.1 \%$ \\
\hline 0.000 & 36.8777 & 36.8675 & $0.0 \%$ \\
\hline 0.005 & 36.8390 & 36.8666 & $-0.1 \%$ \\
\hline 0.010 & 36.7295 & 36.4168 & $0.9 \%$ \\
\hline 0.015 & 36.5951 & 35.9671 & $1.7 \%$ \\
\hline 0.020 & 36.4870 & 35.9258 & $1.5 \%$ \\
\hline 0.025 & 36.4216 & 35.8844 & $1.5 \%$ \\
\hline 0.030 & 36.4108 & 35.9497 & $1.3 \%$ \\
\hline 0.035 & 36.4937 & 36.0150 & $1.3 \%$ \\
\hline
\end{tabular}

It can be seen that there is a small difference in the results which may be due to the fact that the discretization in $3 \mathrm{D}$ is slightly less refined than in $2 \mathrm{D}$.

Next the formulation of the Genetic Algorithm which determines the location and the size of the tumour is presented.

\section{OPTIMIZATION ALGORITHM}

Genetic Algorithms are a family of computational models inspired by the natural selection of living beings. These models are created to follow the laws of natural selection guaranteeing the evolution of a given population of individuals (Castro and Partridge, 2006).

Considering five characteristics cited by Lemonge and Barbosa (2004), the Genetic Algorithm employed can be described as follows:

\subsection{Genetic representation adopted}

Binary Coding, with Chromosomes of 14 digits. These digits have the following meaning:

- 4 digits to represent the $\mathrm{x}$ coordinate, one has then $n p=2^{4}$, or 16 possible solutions for the $\mathrm{x}$ coordinate mapped on the interval $[-0.03 ; 0.03]$ in meters, guaranteeing an accuracy of de $+/-1.875 \mathrm{~mm}$ in the results.

- 3 digits for the y coordinate which gives 8 possible solutions, mapped on the interval $[0.002 ; 0.026]$ guaranteeing an accuracy of $1.5 \mathrm{~mm}$ in $\mathrm{y}$.

- 4 digits to represent the $\mathrm{z}$ coordinate in such a way that there are 16 possible solutions for the $\mathrm{z}$ coordinate, mapped on the interval $[-0.03 ; 0.03]$ in meters thus giving an accuracy of $+/-1.875 \mathrm{~mm}$ in the results.

- 3 digits for the size, in such a way that there are 8 possible sizes of rectangular tumour mapped on the interval $[0.25 ; 2.00]$ where the sizes are proportional to the dimensions 
0.02 in $\mathrm{x}, 0.01$ in $\mathrm{y}$ and 0.02 in $\mathrm{z}$. Thus a tumour described as of dimensions $(0.02 ; 0.01$; $0.02)$ has size 1.00 in the mapping described. A similar scheme was adopted for the spherical models. For the spherical models, a sphere of size 1.00 is a sphere of diameter $0.02 \mathrm{~m}$

The chromosome has thus 4 alleles. Thus the 14 digits make possible 16384 different solutions, this then is the size of the search space, and only one of these will be given as an ideal solution. The search space comprises the whole of the region defined above. If the chromosome indicates a tumour which is in a position in which part of the tumour would be outside the search space, this will be automatically eliminated by the fitness function because the temperature distribution will be discontinuous and completely different from a real distribution.

\subsection{Initial population}

The initial population is created using a subroutine for generating random numbers, (Press et al, 1986), in such a way that each digit has a $50 \%$ possibility of taking a given binary value (0 or 1$)$. A population with 25 individuals is adopted.

\subsection{Fitness function}

The objective function should be capable of minimizing the value of the sum of the squares of the differences between the temperatures calculated on surface in ABCD (Fig. 2) and the values of the temperature distribution obtained previously and which correspond to the position and the size of the tumour which it is desired to locate. The chromosomes are put into reverse order according to the value of the objective function and the values of the fitness function are obtained by linear scaling.

\subsection{Genetic operators}

The individuals which have a greater probability of selection according to the fitness function are selected for crossover, using the roulette wheel method described by Goldberg (1989). Crossover using between four and eight points is carried out between the pairs of chromosomes selected, being one or two per allele.

A process of elitism is employed where the two best individuals in a generation are passed automatically to the next assuring that the best solution is not lost.

\subsection{Stopping criteria}

Two criteria for stopping were defined:

- Criterion number 1: a maximum of 60 generations is permitted. If the process gets to the last generation, calculations are interrupted and the algorithm returns the best solution obtained up to that moment;

- Criterion number 2: The algorithm will start looping after exploring all of the possible solutions near the best solution obtained, as defined by the function "near search" (to be considered below).

Latin American Journal of Solids and Structures 10(2013) 1061 - 1079 
For the Genetic Algorithm used here, in addition to the characteristics common to the majority of such algorithms, new functions were proposed to improve the performance of the same, given difficulties found in obtaining a solution in some cases. In this way the functions "history" and "near search" were created and which are described next.

\subsection{History function}

In order to avoid repetitive calculations, which arise in the execution of a Genetic Algorithm, a function was created which stores a history of all of the individuals which have been created up to that moment, and before the calculation of the objective function a new individual is submitted to this history. If this individual has a predecessor with the same genetic information, the calculation of the objective function is omitted and the value is attributed which was obtained for the identical predecessor.

The history function also contributes a new possibility of evaluating the efficiency of the algorithm, because it is known that the search space is finite. One form of finding an ideal solution would be to evaluate all of the possible solutions; however this is not viable because of the large number of solutions. In this way, a knowledge of how many individuals were calculated during the optimization process is a good indication of the efficiency of the method.

\subsection{Local search function}

As has been said above, crossover is one of the principal search processes in a Genetic Algorithm. And this is greatly responsible for exploring unknown regions of the search space. One criticism that can be made of the crossover algorithm is that if two parent individuals contain the same genetic information, two individuals identical to the parents will be generated; this is common in the final stages of the execution of a genetic algorithm. These algorithms generally stop because most of the individuals in the last generation have the same genetic characteristics.

In the first generations this problem is not very perceptible; up to then a small selection pressure is enough to solve the problem, however in the last generations the problem is far more serious. A refined search might not be possible, because the lack of genetic information might indicate a local maximum as the problem solution. Another important fact is that in situations where the function to be optimized has oscillations near the maximum point, the lack of genetic information can again cause the algorithm to select a solution near to the maximum point and not find the true solution exactly.

To solve these two problems the following is proposed: whenever an individual recently created by crossover is identical to an individual that has already existed, (this can be seen by applying the history function), this is substituted by an unused change to the best individual generated up to that moment.

The effect that will be inserted in the Genetic Algorithm is that of a search near the maximum point, preventing convergence up to the point in which all of the points near the best result have been analysed. At this point the algorithm will start to loop because it is no longer possible to find unused individuals near the best solution. In this way the maximum number of attempts to 
generate an unused individual should be fixed. One has here a new stopping criterion. Here a maximum of 1000 attempts were considered.

The distance considered in the proximity of the optimum point should be monitored in order that the algorithm does not find just any point in the search space, which might be far from the region being investigated. A maximum distance should be fixed in which the search will be concentrated. The maximum distance used here is 1 position for the variables y and "size" which use alleles of three digits and two positions for the variables $\mathrm{x}$ and $\mathrm{z}$ which use alleles of 4 binary digits. By position here it is meant one or two binary intervals as defined in section 4.1. This function is only used intensively when the algorithm initiates the process of convergence. In this way the "local search" function does not diminish the search capacity of the Genetic Algorithm but adds at the final stage a capacity for refining the solution, in addition to reducing the large quantities of repeated calculations that the Genetic Algorithm executes in the final stage of convergence.

\section{RESULTS}

\subsection{Inverse problem in the general formulation}

\section{Case 1: Rectangular Tumour, $0.02 \times 0.01 \times 0.02 \mathrm{~m}$, centred at $(0.00 ; 0.01 ; 0.00) \mathrm{m}$.}

After 6 generations it was already possible to observe the presence of the ideal solution amongst the 25 individuals in the population, as a consequence this solution was present in all the future generations. The algorithm stopped after 51 generations because stopping criterion 2 had been reached. The history function counter indicated that 951 possible solutions of a total of 16384 had been calculated, which shows the efficiency of the search method.

The solution found was a tumour with dimensions $0.02 \times 0.01 \times 0.2 \mathrm{~m}$ centred on $(0.0 ; 0.11$; 0.00), which produced the temperature distribution given in fig. 7 (found), compared with the original distribution (expected).

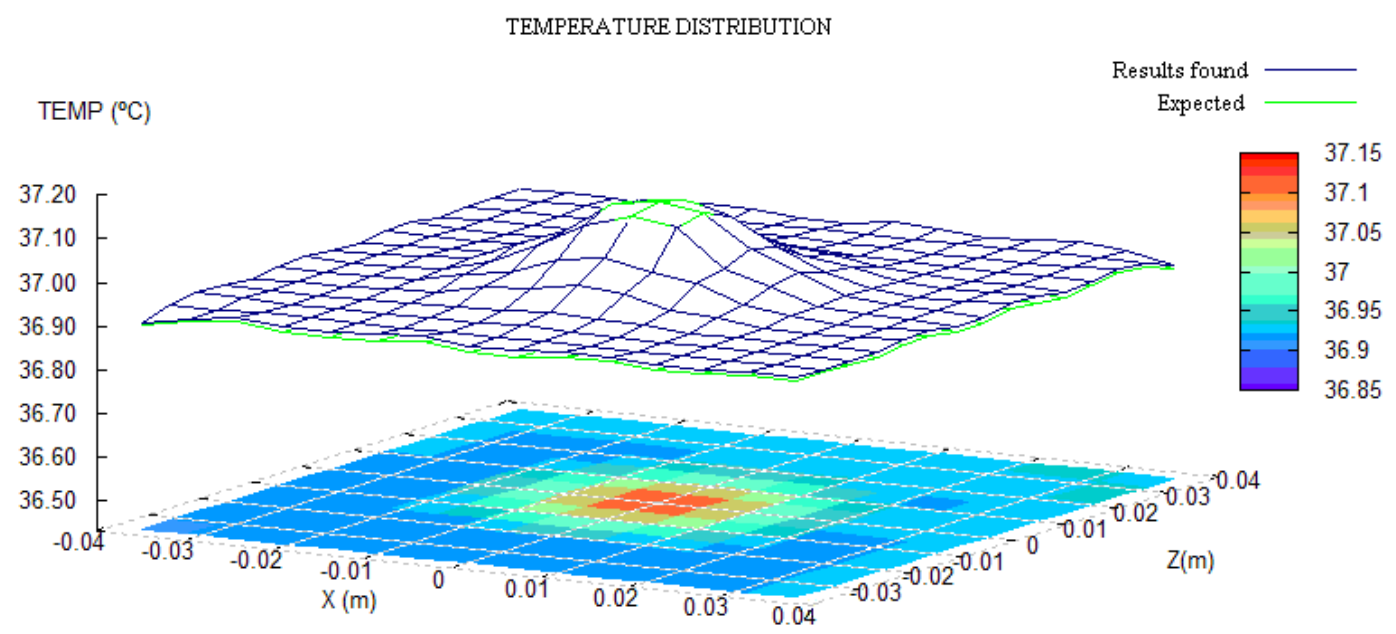

Figure 7 Comparison of the temperature distributions found and expected for case 1 . 
It can be seen that there is a small difference between the results found and those expected, due to the fact that the binary mapping of the variables is not capable of representing exactly the expected value. The accuracy of the approach is discussed above. However it can be noted that this small difference does not affect the quality of the solution found.

\section{Case 2: Rectangular tumour, $0.03 \times 0.015 \times 0.03 \mathrm{~m}$ centred at $(0.01125 ; 0.0011 ; 0.01125) \mathrm{m}$.}

In this case 40 generations were necessary for the algorithm to find the ideal solution. Calculations ceased after 50 generations because stopping criterion 2 was reached with a total of 903 individuals analysed.

The solution found was a tumour with dimensions equal to those expected which could be represented exactly. The dimensions were $0.03 \times 0.015 \times 0.03 \mathrm{~m}$ centred at $(0.01125 ; 0.0011$; $0.01125)$.

\section{Case 3: Spherical tumour, diameter $0.02 \mathrm{~m}$, centred at $(0.00 ; 0.01 ; 0.00) \mathrm{m}$.}

The ideal solution appeared in the population after 12 generations. The algorithm stopped after 60 generations because stopping criterion 1 was reached (Maximum number of generations). The counter in the History Function indicated that 1119 possible solutions of a total of 16384 were calculated. The solution found was a tumour with a diameter of $0.02 \mathrm{~m}$ centred on $(0.00 ; 0.011$; 0.00 ), which produced the temperature distribution shown in fig. 8 in which the distribution found is compared with the expected distribution.

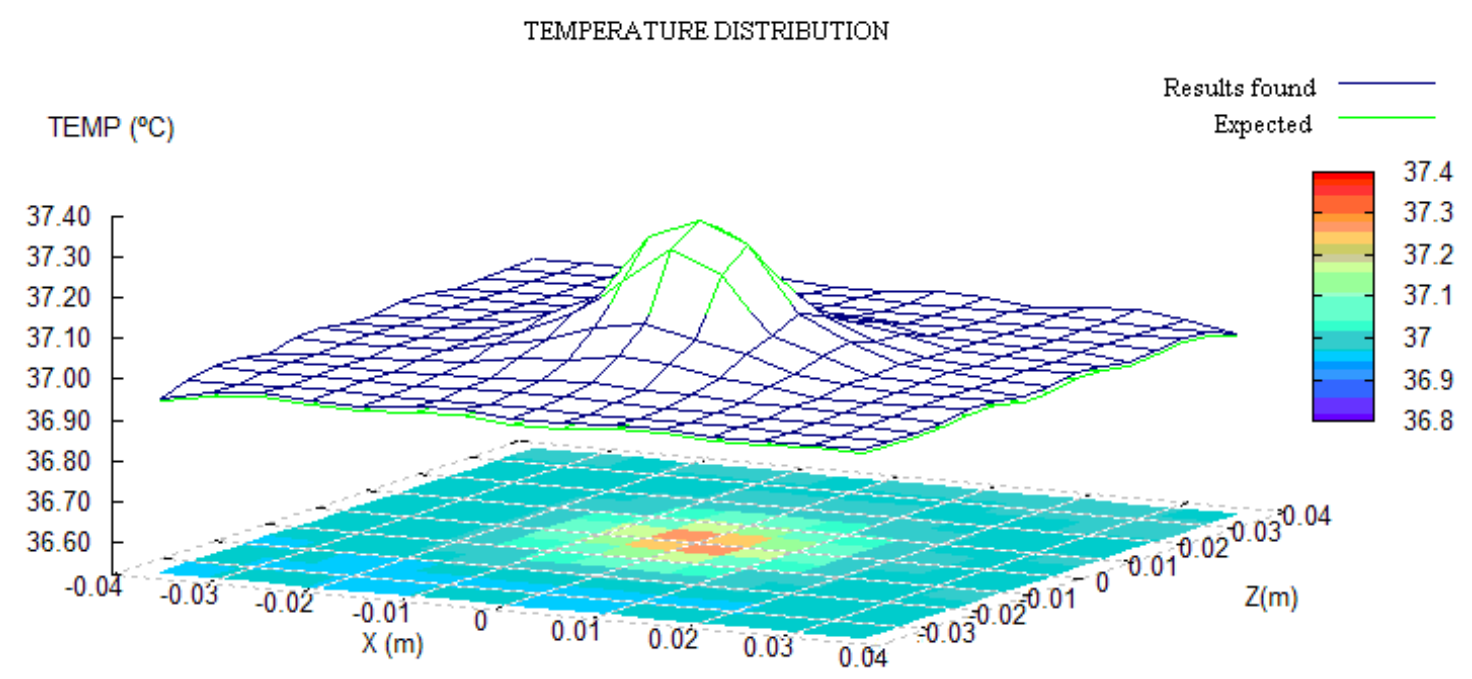

Figure 8 Comparison of found and expected temperature distributions for case 3.

\section{Case 4: Spherical tumour diameter $0.02 \mathrm{~m}$, centred at $(-0.015 ; 0.02 ; 0.01125) \mathrm{m}$.}

The solution appeared in the population after 19 generations. Calculations ceased after 46 generations when stopping criterion 2 was reached, the History Function showed that 830 individuals 
had been analysed. The solution found was a tumour with the dimensions expected which could be represented exactly.

\subsection{Cases considering convection and radiation}

Convection and radiation can be considered together in order to obtain a more realistic boundary condition when the skin surface is exposed.

\section{Case 5: Rectangular tumour, $0.035 \times 0.0175 \times 0.035 \mathrm{~m}$ centred at $(0.00 ; 0.02 ; 0.0125) \mathrm{m}$.}

The ideal solution appeared amongst the population after 24 generations. Calculations ceased after 43 generations because stopping criterion 2 was reached with a total of 783 individuals analysed. The solution found was a tumour with dimensions exactly equal to those expected.

\section{Case 6: Spherical tumour, diameter $0.015 \mathrm{~m}$ centred at $(0.00375 ; 0.008 ; 0.0075) \mathrm{m}$.}

After 24 generations the presence of the ideal solution could be observed amongst the individuals of the population. Calculations ceased after 38 generations because stopping criterion 2 was reached after the whole of the neighbourhood of the optimum point had been explored with the analysis of 698 individuals. The solution found was identical to that expected.

\subsection{Consideration of errors in measurements}

Equipment for measuring temperature on the skin surface was studied in the medical literature by (Togawa, 1985). It is known that such equipment has an error of the order of $0.05{ }^{\circ} \mathrm{C}$ for older equipment and $0.04{ }^{\circ} \mathrm{C}$ for commercial quartz crystal oscillators, being as little as $0.02{ }^{\circ} \mathrm{C}$ for modern equipment using infrared radiation.

To simulate temperature measurement on the skin surface and evaluate the capacity of the Genetic Algorithm to take in to account the accuracy of existing equipment, errors were introduced into the observed temperature data in a random way (Press et al, 1986) The standard deviation was monitored in such a way that the most accurate readings had an error of $0.01{ }^{\circ} \mathrm{C}$ and the least accurate $0.06{ }^{\circ} \mathrm{C}$.

Case 7 : Rectangular tumour $0.02 \times 0.01 \times 0.02 \mathrm{~m}$ centred at $(0.00 ; 0.011 ; 0.00)$, measurement error $\sigma=0.0267$ o. C.

After 26 generations the presence of the solution can be observed amongst the 25 individuals of the population. Calculations ceased after 55 generations because stopping criterion 2 was reached. The counter in the history function indicates that 955 individuals of a possible 16384 were calculated. The solution found was a tumour with dimensions equal to those expected. The temperature distributions are shown in fig. 9 in which the results found are compared with the expected values.

Latin American Journal of Solids and Structures 10(2013) $1061-1079$ 


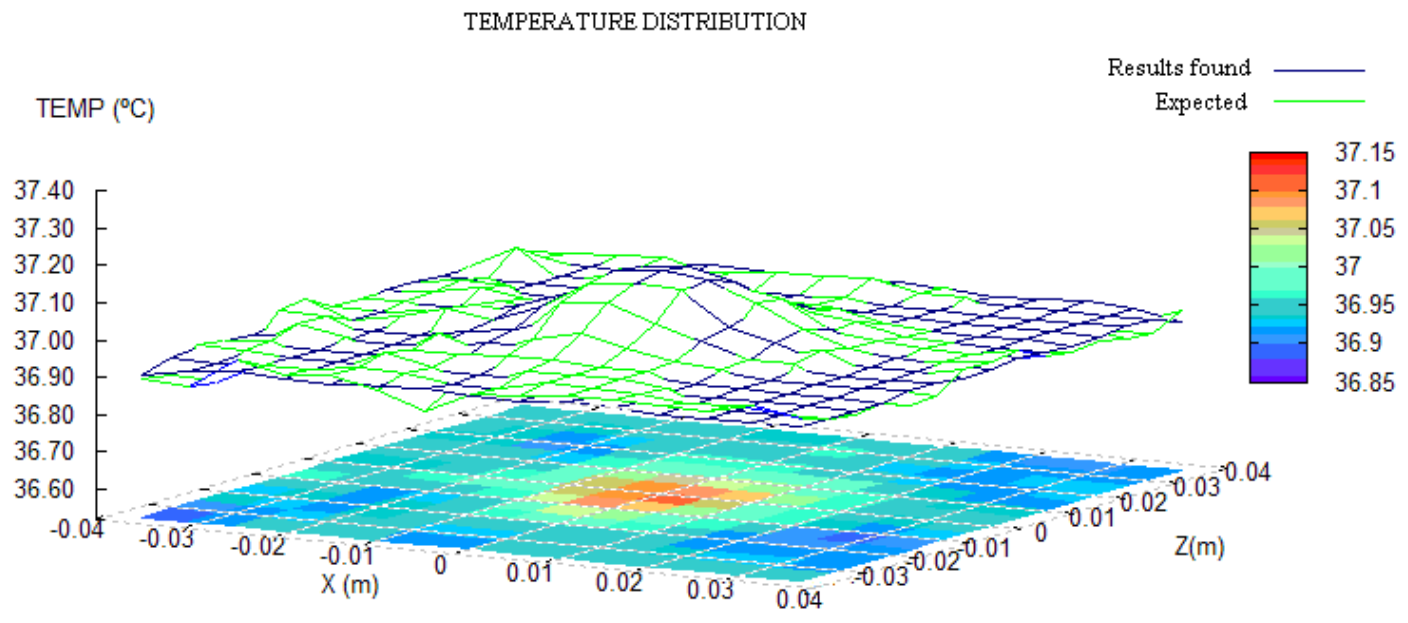

Figure 9 Comparison between the found and expected temperature distributions for case 7.

Case 8: Spherical tumour, diameter $0.015 \mathrm{~m}$, centred at $(0.00375 ; 0.008 ; 0.0075)$, standard deviation $\sigma=0.0178 \stackrel{\mathrm{o}}{ }$, considering convection and radiation

The solution appeared amongst the population after 6 generations. Calculations ceased after 41 generations when stopping criterion 2 was reached. The counter in the history function indicated that 760 possible solutions were calculated of a total of 16384 . The tumour found had dimensions equal to those expected.

\section{CONCLUSIONS}

\subsection{Discussion}

In cases 1 and 3 the difference in the results for the y coordinate is due to the binary mapping which, in the way in which it was defined, has an accuracy of $0.0015 \mathrm{~m}$.

The Genetic Algorithm may find the solution to the problem in the first few generations, as happens for example, in case 1, where the solution appears amongst the population at generation 6 . However to guarantee that this really is the best solution it is necessary to execute more generations. Calculations stopped at generation 51. The number of generations was small confirming the correct dimensioning of the Genetic Algorithm.

The addition of noise to the input data, with the intention of simulating imperfections in the equipment used for measuring the temperatures, did not alter the capacity of the algorithm to find accurate solutions (cases 7 and 8).

The implementation of boundary conditions in convection and radiation associated with the addition of noise to the input data did not generate interference in the results obtained in Case 8, given that this example considers the same tumour as case 6 and in both cases the same solution was obtained even with the same errors due to the mapping of variables. 


\subsection{Conclusions}

The Boundary Element Method shows itself to be viable for the solution of inverse problems with the Genetic Algorithm in 3D. It should be noted that the method does not need to remesh for each new individual considered as would be the case with domain methods.

The Genetic Algorithm in its traditional form carries out many repeated calculations which is a limiting factor in the case of DRBEM. This problem was tackled by the addition of functions which avoid the reprocessing of individuals which have been calculated before and effort was concentrated on searching for possible solutions in the vicinity of the best solution found up to the moment. These routines permitted a large reduction in processing time and also permitted the algorithm to succeed in some cases where it would have failed.

A population of 25 individuals was only possible for a problem with 4 variables due to the efficiency of the functions "history" and "near search" developed here.

Thermal methods are an alternative for aiding health workers in the diagnosis of tumours and also show themselves to be a solution for accompanying the treatment of the same.

The Genetic Algorithm used here was developed for the problem of the localization of tumours; however the same can be used with other types of optimization problems. The functions implemented can bring benefits for the general use of the Genetic Algorithm.

\section{References}

[1] Wrobel, L. C.; PARtridge, P. W.; De CAStro, L. C. L. B.; BUeno, F. R. A Bem technique for the detection of skin tumours by thermal analysis. In: UK Conference on boundary integral methods, 7, Proceeding. Nottingham, p. 99 - 106, 2009.

[2] BUENO, F. R.; PARTRIDGE, P. W. A study of dual reciprocity for three dimensional models applied to the solution of Pennes Bioheat equation. In: Beteq, XII, 2011, Proceedings... Brasilia, p. 182-189, 2011.

[3] CHAN, C. L. Boundary element method analysis of the bioheat transfer equation. Journal of Biomechanical Engineering, v. 114, p. 358-365, 1992.

[4] De CASTRO, L. C. L. B.; PARTRIDGE, P. W. Minimum weight design of framed structures using a genetic algorithm considering dynamic analysis. Latin American Journal of Solids and structures, v. 3, p. 107-123, 2006.

[5] DENG, Z. S.; LIU, J. Modelling of multidimensional freezing problem during cryosurgery by the dual reciprocity boundary element method. Engineering Analysis with Boundary Elements, v. 28, p. 97-108, 2004.

[6] DENG, Z. S.; LIU, J. Parametric studies on the phase shift method to measure the blood perfusion of biological bodies. Medical Engineering $\& 3$ Physics, v. 22, p. 693-702, 2000.

Latin American Journal of Solids and Structures 10(2013) $1061-1079$ 
[7] GOLDBERG, D. E. Genetic algorithms in search, optimization and machine learning. $1^{\text {st }}$ ed. Boston: Addison Wesley Longman Publishing Co., 1989.

[8] Lemonge, A. C. C.; BARBOSA, H. J. C. An adaptive penalty scheme for genetic algorithms in structural optimization. International Journal for Numerical Methods In Engineering, v. 59, p. 703-736, 2004.

[9] LIU, J.; XU, L. X. Boundary information based diagnostics on the thermal states of biological bodies. International Journal of Heat and Mass Transfer, v. 43, p. 2827-2839, 2000.

[10] PARTRIDGE, P. W.; WROBEL, L. An inverse geometry problem for the localisation of skin tumours by thermal analysis. Engineering Analysis with Boundary Elements, v. 31, p. 803$811,2007$.

[11] PARTRIDGE, P. W.; WROBEL, L. C; BREBBIA, C. A. The dual reciprocity boundary element method. London: Elsevier Applied Science; Southampton: Computational Mechanics Publications, 1992.

[12] PENNES, H. H. Analysis of tissue and arterial blood temperatures in the resting human forearm. Journal of Applied Physiology, v. 1, n. 2, p. 93-122, 1948.

[13] PRESS, W. H., FlAnNeRY B. P.; TEUKOlSKY, S. A.; VETTERling, W. T. Numerical recipes: the art of scientific computing. $1^{\text {st }}$ ed. Cambridge: Cambridge University Press, 1986.

[14] TOGAWA, T. Body temperature measurement. Clinical Physics and Physiological Measurement. v. 6, n. 2, p. 83-108, 1985.

[15] WISSLER, E. H. Pennes' 1948 paper revisited. Journal of Applied Physiology, v. 85, p. 3541, 1998.

[16] WISSLER, E. H. Steady-state temperature distribution in man. Journal of Applied Physiology, v. 16, p. $734-740,1961$. 
